Dear author,

Please note that changes made in the online proofing system will be added to the article before publication but are not reflected in this PDF.

We also ask that this file not be used for submitting corrections. 
Research paper

\title{
Analysis of the influence of coordinate and dynamic formulations on solving biomechanical optimal control problems
}

\author{
Gil Serrancolí*, Rosa Pàmies-Vilà \\ Department of Mechanical Engineering, Universitat Politècnica de Catalunya, Av. Eduard Maristany 16, 08019 Barcelona, Catalunya, Spain
}

\section{A R T I C L E I N F O}

Article history:

Received 21 February 2019

Revised 23 July 2019

Accepted 24 July 2019

Available online $\mathrm{xxx}$

\section{Keywords:}

Optimal control problem

Multibody formulation

Ill-conditioning

Mass matrix

Hessian matrix

Predictive simulation

\begin{abstract}
A B S T R A C T
Predictive simulations are useful in biomechanics to determine the optimal trajectories between two mechanical states and their forces in an in-silico approach. To perform these predictions, optimal control problems are increasingly in use. However, the efficiency of their formulation is crucial: in order to converge to the same optimal solution, some formulations could take a higher number of iterations than others. The paper presents an analysis of how certain key factors affect the convergence of these simulations. We compare the convergence of several optimal control problems solved using a collocation method. Eight planar torque-driven models of a human skeleton are used with four different types of coordinates and two dynamic formulations. The results show that both the mass matrix of the multibody system and the Hessian matrix of the non-linear problem affect the convergence of the solution: there is a statistical significant relationship among the number of iterations and the conditioning of these two matrices. Moreover, the study shows that the optimal control problems formulated with absolute coordinates are the ones requiring the lowest number of iterations overall. These findings suggest that formulations leading to poor conditioning of mass and Hessian matrices should be avoided.
\end{abstract}

(c) 2019 Elsevier Ltd. All rights reserved.

\section{Introduction}

Over the last decades, the prediction of human motion has been a subject of intense research among the biomechanical scientific-technical community. These techniques may have potential clinical applications, such as predicting how a specific person will move after a surgery, customizing assistive devices, or testing theories of movement control [1-5]. However, the practical use of these techniques is still a challenge, and nowadays their clinical applications are still incipient.

In general, the methods developed for this purpose can be classified in accordance with the type of human model (torque or muscle-driven model), the design variables chosen, or whether some variables are tracked or not. Some authors used a muscle model to solve the muscle redundancy problem from inverse dynamics joint torques of a given movement $[6,7]$. Other authors used a torque-driven model to predict both kinematics and joint torques simultaneously [8-10]. Muscledriven models have also been used to predict kinematics and muscle activation simultaneously [11-19]. In addition, some authors tracked experimental joint coordinates, torques, ground reaction forces or muscle activations to calibrate a neuromusculoskeletal model, or simply to find a dynamic consistent motion that fits the measured data [20-23].

\footnotetext{
* Corresponding author.

E-mail address: gil.serrancoli@upc.edu (G. Serrancolí).
} 
The aforementioned studies have in common that they formulated optimal control problems to perform their simulations. This technique has become popular over the recent years in biomechanical motion prediction mainly due to an increase of computational capacities and the development of new optimization software. Optimal control problems for biomechanical analysis involve an objective function, usually expressed as an integral of a function over a period of time, the so-called functional. This functional represents some physical quantity, such as the metabolic energy cost, or the sum of squared torques, joint accelerations or muscle activations [24-26].

Optimal control problems are computationally expensive and not straightforward to solve due to the stiffness and nonlinearity of the differential equations. Different numerical methods have been proposed to solve these problems [27]. Direct collocation is one of the most used methods in predictive dynamics [19,28-30]. As the previous cited studies show, there are various ways to formulate the optimal control problems (different coordinates, dynamic formulations or degrees of complexity). Consequently, comparing the results in terms of convergence efficiency is not suitable when several formulations are mixed. Therefore, the goal of this study is to analyze the influence of the dynamic formulations, the coordinates and the complexity of the models on the performance of the optimizer.

When direct collocation methods are used, the optimization problem is approximated as a highly sparse nonlinear programming (NLP) problem and it can be solved efficiently using gradient-based NLP solvers. There are two matrices of particular interest when using this formulation: the Hessian matrix of the NLP problem, which contains second order derivatives of the Lagrange variable (objective function and constraints of the NLP problem); and the mass matrix of the system, which contains the inertial properties. The convergence of the optimal control problem might be influenced by these matrices, which in turn depend on the type of coordinates used to describe the model and the dynamic formulation used to introduce the equations of motion.

On the one hand, when an explicit formulation is used, it is important to perform an analysis of the multibody mass matrix before solving optimal control problems, since the matrix needs to be inverted. In these cases, ill-conditioned mass matrices could make the convergence of the optimization difficult. Therefore, one could consider that multibody formulations with constant mass matrices may lead to better convergences. In this study, one of the tested coordinates are the natural coordinates (Cartesian coordinates), which lead to constant mass matrices (positive definite [31,32]). The conditioning of the mass matrix might also be influenced by the starting point of the kinematic chain, especially when using relative coordinates. On the other hand, the conditioning of the Hessian matrix of the NLP problem caused by the use of different coordinate systems or dynamic formulations (explicit or implicit) could also have an impact on the convergence results.

This work compares the behavior of the convergence of optimal control problems when minimum and non-minimum coordinates are used, and when using explicit or implicit dynamic formulations. For this purpose, the number of iterations to find an optimal solution and the conditioning of both the mass matrix of the multibody system and the Hessian of the NLP problem are used as indicators. The mass matrix is studied through its condition number [33,34], which reveals the numerical "illness degree" of a matrix, and can be used to evaluate the sensitivity of a linear system subjected to some disturbance [35]. In the proposed work, the mass matrix cannot be singular, but ill-conditioned depending on the formulation used. In contrast, the Hessian of the NLP problem is rank-deficient if the design variables are not all included in a non-linear form within the objective or constraint function. For this reason, we also use the normalized rank of the Hessian as a parameter to explain the variability of the optimization convergence. In this study eight planar skeleton models (from 3 to $10 \mathrm{DoF}$ ) are formulated using absolute, relative and natural coordinates. For each model and type of coordinate definition, an optimal control problem is solved twice: using implicit and explicit dynamic formulations. Each problem consists in predicting a swing phase of human gait from an initial to a final state minimizing the integral of squared joint torque values.

\section{Methods}

\subsection{Biomechanical models}

Eight torque-driven planar models of increasing complexity are used to predict the swing phase of human gait using different types of coordinates. All models have the right foot attached to the ground and ranged from 3 to 10 DoF. As shown in Fig. 1, the initial model is a 3-DoF model with ankle joint (right) and hip joints (right and left), then complexity increases up to a 10-DoF model with neck joint, ankle joint (right) and knee, hip, shoulder and elbow joints (right and left). Mass and inertia of the segments are modified according to their dimensions, preserving a total mass of $83 \mathrm{~kg}$ and a height of $1.67 \mathrm{~m}$ for all models. The equations of motion are obtained applying Lagrange equations using the symbolic toolbox in MATLAB.

To compare the performance of optimal control problems predicting the swing phase, we use different types of coordinates of the multibody model: absolute, natural and relative coordinates (Fig. 2). The set of absolute coordinates is defined as angles between the horizontal axis and each segment (Fig. 2a). The set of natural coordinates is defined as the Cartesian coordinates of each joint (Fig. 2b). Two sets of relative coordinates are tested, defined as relative angles among consecutive bodies of the kinematic chain: in one set the kinematic chain starts at the hip (Fig. 2c) and in the other set it starts at the right ankle, which is fixed to the ground (Fig. 2d).

The main difference when using natural coordinates instead of absolute or relative coordinates is related to the mass matrix and the constraints that impose consistency of segment lengths. On the one hand, it is known that the multibody formulation using relative and absolute coordinates leads to a non-constant mass matrix, which depends on the value of the 


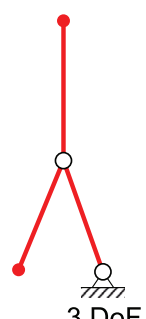

3 DoF

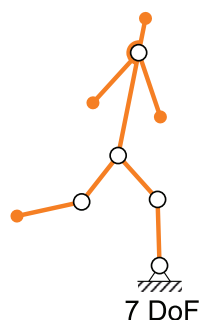

$7 \mathrm{DoF}$

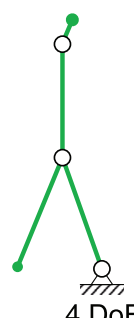

$4 \mathrm{DoF}$

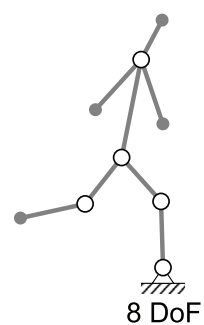

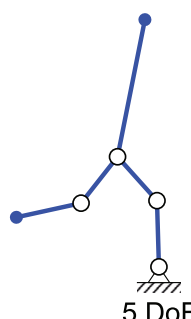

5 DoF

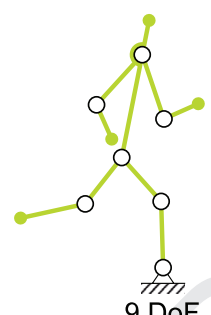

$9 \mathrm{DoF}$

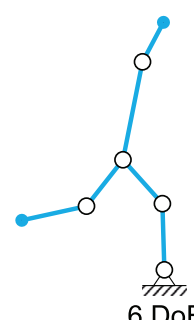

$6 \mathrm{DoF}$

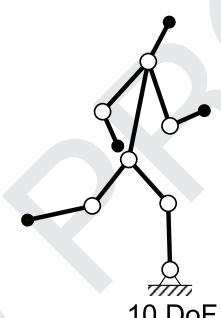

Fig. 1. Humanoid planar models from 3 to 10 DoF. Each circle represents a hinge joint. A circular arc around the neck joint means that the head is fixed to the trunk.

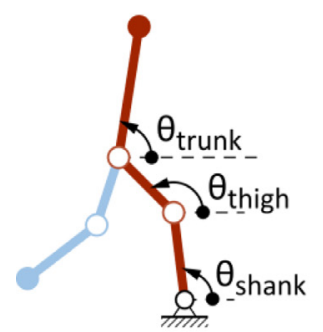

Absolute

(a)

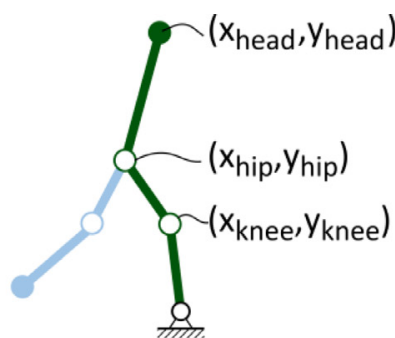

Natural

(b)

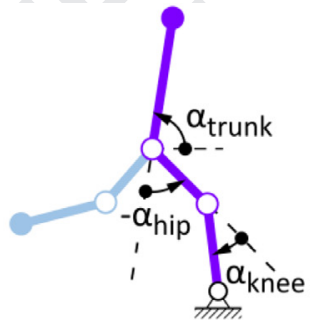

Relative from hip

(c)

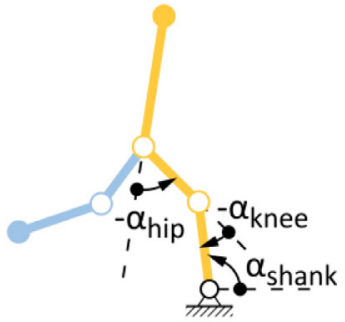

Relative from ankle

(d)

Fig. 2. Types of coordinates used to represent the multibody model. (a) absolute coordinates; (b) natural coordinates; (c) relative coordinates starting the kinematic chain from the hip joint; (d) relative coordinates starting the kinematic chain from the ankle joint. Only some coordinates are mentioned to illustrate a generic case.

coordinates [36]. On the other hand, the use of natural coordinates leads to a constant mass matrix (independent of position and orientation) $[28,32,37]$. Moreover, in the latter case, the Lagrange variable $(L)$ takes into account that the lengths of the segments are constant:

$$
L=T-U+\sum_{i}^{n c} \lambda_{i} c_{i}(\mathbf{q})
$$

where $T$ and $U$ are the kinetic and the potential energy, respectively; $\mathbf{q}$ is the vector of generalized coordinates, $n c$ is the number of coordinates, and $\lambda$ are the Lagrange multipliers. In this case, $c_{i}(\mathbf{q})$ is the difference between the squared distance of the extreme points of each segment of the model and its defined squared length [38]. For example, for segment 1, with length $l_{1}$, and extreme points $\mathrm{P}_{1}=\left(x_{1}, y_{1}\right)$ and $\mathrm{P}_{2}=\left(x_{2}, y_{2}\right)$ :

$$
c_{1}(\mathbf{q})=\left(x_{1}-x_{2}\right)^{2}+\left(y_{1}-y_{2}\right)^{2}-l_{1}^{2}
$$

As mentioned, the conditioning of the mass matrix could have an influence on the optimal control problem formulation, especially if this matrix needs to be inverted. In turn, the conditioning of the mass matrix using relative coordinates might depend on the initial point of the kinematic chain of the multibody model. For this reason, we use the two mentioned sets of models in relative coordinates (from 3 DoF to 10 DoF) starting from two different joints. The set of models starting the kinematic chain from the hip has extra coordinates (Cartesian coordinates) to define the position of the pelvis joint with respect to the ground. In this case, the extended Lagrange variable (Eq. (1)) contained a constraint to keep the right foot attached to the ground and fixed to the origin:

$$
c(\mathbf{q})=\left[x_{\text {right foot }}(\mathbf{q}), y_{\text {right foot }}(\mathbf{q})\right]
$$

where $x_{\text {right foot }}(\mathbf{q})$ and $y_{\text {right foot }}(\mathbf{q})$ are the horizontal and vertical positions of the right foot with respect to the ground. 


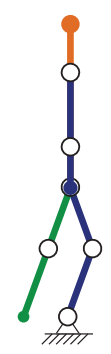

$t=t_{0}$
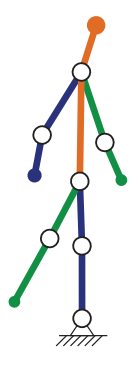
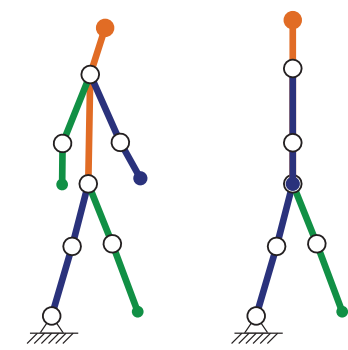

$t=t_{\mathrm{f}}$

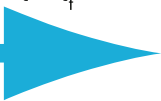

Fig. 3. Representation of the initial (left) and the final (right) state configurations of the swing predicted motion with two intermediate states.

\subsection{Optimization algorithm}

An optimal control problem using a direct collocation approach is formulated to predict the kinematic and dynamic evolution from an initial to a final state in $0.5 \mathrm{~s}$ for all models. This movement represents the left leg swing phase, as shown in Fig. 3. The right foot is considered to be fixed to the ground (single support phase). The initial state represents the beginning of the swing phase in rest (zero velocities), just after the left foot is detached from the ground. The final state represents the state just before the left foot contacts the ground (with free velocities). The minimization criterion is the same for all optimizations: minimizing the integral value (functional) of the squared values of joint torques $\left(u_{T}\right)$ :

$$
J=\int_{t_{0}}^{t_{f}} \sum_{i}^{n D o F} u_{T_{i}}^{2} d t
$$

In all optimal control problems, the state variables are the coordinates and their velocities:

$$
\mathbf{x}_{\text {Ab }}=\left[\theta_{\text {shank }}, \ldots, \dot{\theta}_{\text {shank }}, \ldots\right]
$$

$$
\mathbf{x}_{R e}=\left[\alpha_{\text {ankle }}, \ldots, \dot{\alpha}_{\text {ankle }}, \ldots\right]
$$

$$
\mathbf{x}_{N a}=\left[x_{\text {knee }}, y_{\text {knee }}, \ldots, \dot{x}_{\text {knee }}, \dot{y}_{\text {knee }}, \ldots\right]
$$

Note that the number of state variables in absolute and relative coordinates starting the kinematic chain at the ankle is the same. This number is doubled when natural coordinates are used (Table A1, Appendix A). In all problems, we also consider joint torque controls as design variables, one actuating at each joint:

$$
\mathbf{u}_{T}=\left[u_{\text {Tankle }}, \ldots\right]
$$

Constraint multipliers $\left(\lambda_{i}\right)$ are also included as design variables when natural and relative from hip coordinates are used. In these cases, the equations of motion are calculated with a constrained Lagrange variable (Eq. (1)). The total number of state and control variables and constraint multipliers after discretization are shown in Table A1 (Appendix A).

The direct collocation scheme consists of $N$ mesh intervals with four collocation points at each mesh interval (pseudospectral method [39]). We assume that the state variables follow a parameterization with Lagrange polynomials within each interval, and they are design variables at each collocation point. Control variables are design variables only at the beginning of each time interval, and when these values are requested within a mesh interval, they are interpolated. The benefit of this parameterization is that the cost function, the state and the state derivative values can be calculated at any point of the interval as a function of the state variables at the collocation points [39].

Two optimal control problems are formulated for each model and type of coordinates, one using implicit formulation and the other one using explicit formulation. In explicit formulation, we compute the acceleration of the coordinates from the information of the equations of motion as follows:

$$
\ddot{\mathbf{q}}=[\mathbf{M}]^{-1}(\boldsymbol{\tau}-\boldsymbol{C}(\mathbf{q}, \dot{\mathbf{q}})-\boldsymbol{G}(\mathbf{q}))
$$

where $\ddot{\mathbf{q}}$ are the accelerations, $\mathbf{M}$ is the mass matrix of the multibody system, $\boldsymbol{C}(\mathbf{q}, \dot{\mathbf{q}})$ are the centrifugal terms, $\boldsymbol{G}(\mathbf{q})$ are the gravity terms and $\boldsymbol{\tau}$ are the generalized forces. As mentioned, the inversion of an ill-conditioned mass matrix could have certain influence on the convergence of an optimization. For this reason, the same optimal control problems are tested using an implicit dynamic formulation. In this case, accelerations are modelled as control variables ( $\left.\mathbf{u}_{\mathbf{a}}\right)$ and included as design variables, and the equations of motion are included as path constraints:

$$
[\mathbf{M}] \mathbf{u}_{\mathbf{a}}+\mathbf{C}(\mathbf{q}, \dot{\mathbf{q}})+\mathbf{G}(\mathbf{q})-\boldsymbol{\tau}=0
$$


To ensure state and state derivative continuity, constraints in the NLP problem are included as:

$$
\mathbf{x}_{0}^{i+1}-\tilde{\mathbf{x}}_{\text {end }}^{i}=0
$$

$$
\tilde{\dot{\mathbf{x}}}_{j}^{i}-\dot{\mathbf{x}}_{j}^{i}=0
$$

where $\mathbf{x}_{0}^{i+1}$ are the state variables at the first collocation point of the time interval $i+1 ; \tilde{\mathbf{x}}_{\text {end }}^{i}$ are the state variables at the end of mesh interval $i$, calculated using Lagrange parameterization from the state variable values at the collocation points of its mesh interval; $\tilde{\mathbf{x}}_{j}^{i}$ are the state variable derivatives at collocation point $j$ of mesh interval $i$ calculated using Lagrange parameterization; and $\dot{\mathbf{x}}_{j}^{i}$ are the values of state derivatives at the collocation point $j$ of mesh interval $i$.

We also include boundary conditions and geometrical constraints (depending on the coordinate formulation) to:

- prevent the left foot from penetrating the ground.

- define the range of motion of the neck angle between -450 and 450 in those models containing this joint.

- avoid hyperextension of the knees.

The number of mesh intervals $N$ increases for each model until the same optimal solution is found in all formulations using that model (using all four types of coordinates and both dynamic formulations). The process consists in solving the problems for the same number of DoF starting with $N=25$ intervals. Given the case that one problem does not converge at all or does converge to an optimal solution with a higher optimal cost function value than the other models, we double the number $N$. We repeat this process until all optimal control problems converge to the same optimal solution. We consider the solution to be the same when the root mean square difference of all joint positions with respect to the absolute explicit formulation is lower than $2.0 \mathrm{~mm}$. We also test that the solution does not change (root mean square difference between both solutions lower than $2.0 \mathrm{~mm}$ ) when the value of $N$ from Table I is doubled. The default convergence tolerances might be slightly modified with the same goal, keeping the same tolerance values for models with the same number of DoFs.

In total, 64 optimal control problems are solved in CasADi [40], relying on the optimizer IPOPT [41]. The full sparse Hessian (not approximation) of the Lagrangian variable of the NLP is used to solve the problems. The code of the optimal control problems can be found as Supplementary Material.

\subsection{Analysis}

The goal of this study is to identify the relationship among the performance of the optimizer solving the optimal control problem and the conditioning of both the mass matrix of the multibody system and the Hessian of the NLP problem. To this end, the performance of the optimizer is measured with the number of iterations needed to find an optimal value.

The sensitivity of a linear system $(\mathbf{M x}=\mathbf{b})$ can be measured by the condition number $\left(C N_{\mathbf{M}}\right)$ [33,35]: the higher the condition number of a matrix $\mathbf{M}\left(C N_{\mathbf{M}}\right)$, the higher the change of $\mathbf{x}$ for a certain amount of change of $\mathbf{b}$. In an optimal control problem using an explicit formulation, the computation of the accelerations requires solving a linear system (see Eq. (9)), where matrix $\mathbf{M}$ is the mass matrix of the multibody system. If the condition number of the mass matrix of the multibody system is high, the optimizer may need more iterations to reach an optimal solution than a system with a lower condition number.

Another important factor affecting the convergence of the optimization could be the rank of the Hessian of the Lagrange variable (see Eq. (1)) $\left(\operatorname{Rank}_{\mathbf{H}}\right)$. Since this matrix is used to calculate the search direction at each iteration [41], if the rank of the Hessian is low, the optimizer may also have difficulties in finding the optimal solution with a small number of iterations.

First, the relationship between the difference in number of iterations (implicit minus explicit formulations) and the maximum condition number of the mass matrix is analyzed. Since the value of the condition number can change throughout the movement of the multibody model in relative and absolute formulations, the maximum value of this variable is used.

After that, we investigate the statistical correlation between the performance of the optimal control problems (number of iterations) and the condition number and rank of the Hessian matrix. We use a normalized rank of the Hessian matrix to make the comparisons, i.e., the rank of the matrix is divided by the total number of rows (number of design variables). The statistical correlation is computed using a multiple regression with least squares. We use the F-test in MATLAB to analyze whether the whole regression was significant or not. We also use paired t-tests to analyze whether the number of iterations was significantly different across formulations. The tests are considered significant if p-values are lower than 0.05 .

\section{Results}

\subsection{Number of mesh intervals and modified tolerances}

The number of mesh intervals used to solve the optimal control problems for each model is summarized in Table 1. Note that along this analysis each model is characterized by the number of degrees of freedom. Table 1 results apply equally to all dynamic formulations and coordinates with common DoFs. The joint positions of the optimal solutions found when doubling the number of mesh intervals did not change more than $2.0 \mathrm{~mm}$. 
G. Serrancolí and R. Pàmies-Vilà / Mechanism and Machine Theory xxx (xxxx) xxx

Table 1

Number of mesh intervals $(N)$, and IPOPT tolerances tol, constr_viol_tol, constr_mult_init_max (* stands for default IPOPT values) used to solve the optimal control problem for each model with the same number of DoF.

\begin{tabular}{lllll}
\hline Model (DoF) & Mesh intervals $(\mathrm{N})$ & Tol & Constr_viol_tol & Constr_mult_init_max \\
\hline 3 & 100 & $10^{-4}$ & $10^{-4 *}$ & $1000^{*}$ \\
4 & 100 & $10^{-4}$ & $10^{-4 *}$ & $1000^{*}$ \\
5 & 100 & $10^{-8 *}$ & $10^{-4 *}$ & $1000^{*}$ \\
6 & 400 & $10^{-4}$ & $10^{-4}$ & $10^{4}$ \\
7 & 400 & $10^{-2}$ & $10^{-2}$ & $10^{4}$ \\
8 & 400 & $10^{-2}$ & $10^{-2}$ & $1000^{*}$ \\
9 & 200 & $10^{-2}$ & $10^{-2}$ & $10^{4}$ \\
10 & 200 & $10^{-2}$ & $10^{-4 *}$ & $1000^{*}$ \\
\hline
\end{tabular}

Tolerances regarding the convergence of the algorithm (tol option in IPOPT [41]), the threshold of the constraint violation (constr_viol_tol option in IPOPT) and the maximum allowed least-square guess of constraint multipliers (constr_mult_init_max option in IPOPT) are slightly modified to ensure convergence in all formulations of each model. Formulations with the highest number of constraints, such as natural coordinates and relative coordinates starting the kinematic chain from the hip, are the limiting ones. These values are increased systematically with respect to their default ones; and in each model, they are set to the same values.

\subsection{Optimization performance}

For each model with the same number of DoFs, the results of the optimizations lead to the same optimal solution (see Appendix A), with differences in joint positions with respect to the implicit absolute coordinate formulation lower than $2.0 \mathrm{~mm}$. The number of iterations varies between implicit and explicit dynamic formulations, and among types of coordinates (Table A2, Appendix A). Note that the maximum condition number in implicit and explicit formulations is the same for each type of coordinates, since the mass matrix and the optimal solution are the same.

Fig. 4 shows the results of the maximum condition number of $\mathbf{M}\left(\mathrm{CN}_{\mathbf{M}}^{\max }\right)$ with respect to the difference in the number of iterations between implicit and explicit formulations $\Delta n_{\text {iter }}=N_{\text {imp }}-N_{\text {exp }}$. Red lines show the regression among the solutions from 5 to 10 DoF. Models with 3 and 4 DoF are excluded from the regression since they have more than three times lower condition numbers than the other models. It is important to notice that the regressions do not follow the same tendency across the different types of coordinates. In absolute and relative from ankle type of coordinates, the difference in the number of iterations obtained between implicit and explicit formulations rises when increasing the maximum condition number of the mass matrix. However, in natural and relative from hip type of coordinates the system performs the other way around.

Implicit formulations, which have a lower normalized rank of the Hessian matrix of the NLP problem in this study $\left(\operatorname{Rank}_{\mathbf{H}}^{\text {norm }}\right)$, tend to need more iterations to find an optimal solution (Fig. 5a, Table A2). There is a statistical relationship among the number of iterations, the maximum condition number of the mass matrix and the normalized rank of the Hessian of the NLP problem. The obtained relation is the following:

$$
n_{\text {iter }}=790.78+0.37 C N_{\mathbf{M}}^{\max }-735.86 \operatorname{Rank}_{\mathbf{H}}^{\text {norm }}-0.41 C N_{\mathbf{M}}^{\max } \cdot \operatorname{Ran}_{\mathbf{H}}^{\text {norm }}
$$

which represents a statistically significant relationship, since the p-value is 0.0215 ( $p$-value $<0.05$ ). The same relationship is shown in Fig. $5 b$ highlighting the differences by types of coordinates. The results show that natural coordinates (green) have the lowest values of condition number but they also have the lowest ranks of the Hessian of the NLP problem. This does not lead to a statistically significant decrease in the number of iterations with respect to the other formulations ( $p$-values > 0.05), neither in explicit nor in implicit formulations. When absolute coordinates are used (brown) the rank of the Hessian matrix tends to be the highest (similar to relative coordinates). This type of coordinate also leads to lower values of the maximum condition numbers of mass matrices compared to relative coordinates (yellow and lilac). As a result, the overall lowest values of the number of iterations are obtained using absolute coordinates showing significant differences for the pairs absolute-relative from hip ( $p$-value $=0.04$ in implicit). The projections of the results in the two planes are included in Appendix A, Fig. A1.

\section{Discussion}

The main goal of this study is to analyze the influence of the type of coordinates used in the modeling of a multibody system and the type of dynamic formulation on the convergence of a biomechanical optimal control problem. We have tested the convergence of different optimal control problems with planar models ranging from 3 to 10 DoF, with explicit and implicit dynamic formulations and with different types of coordinates (absolute, relative and natural).

The optimal control problems have been formulated using a direct collocation method, which leads to large-scale NLP problems. These problems have been solved using an interior-point optimizer. The convergence of the optimizations is evaluated comparing the number of iterations needed to reach the optimal solution. The results show that the variability in the 

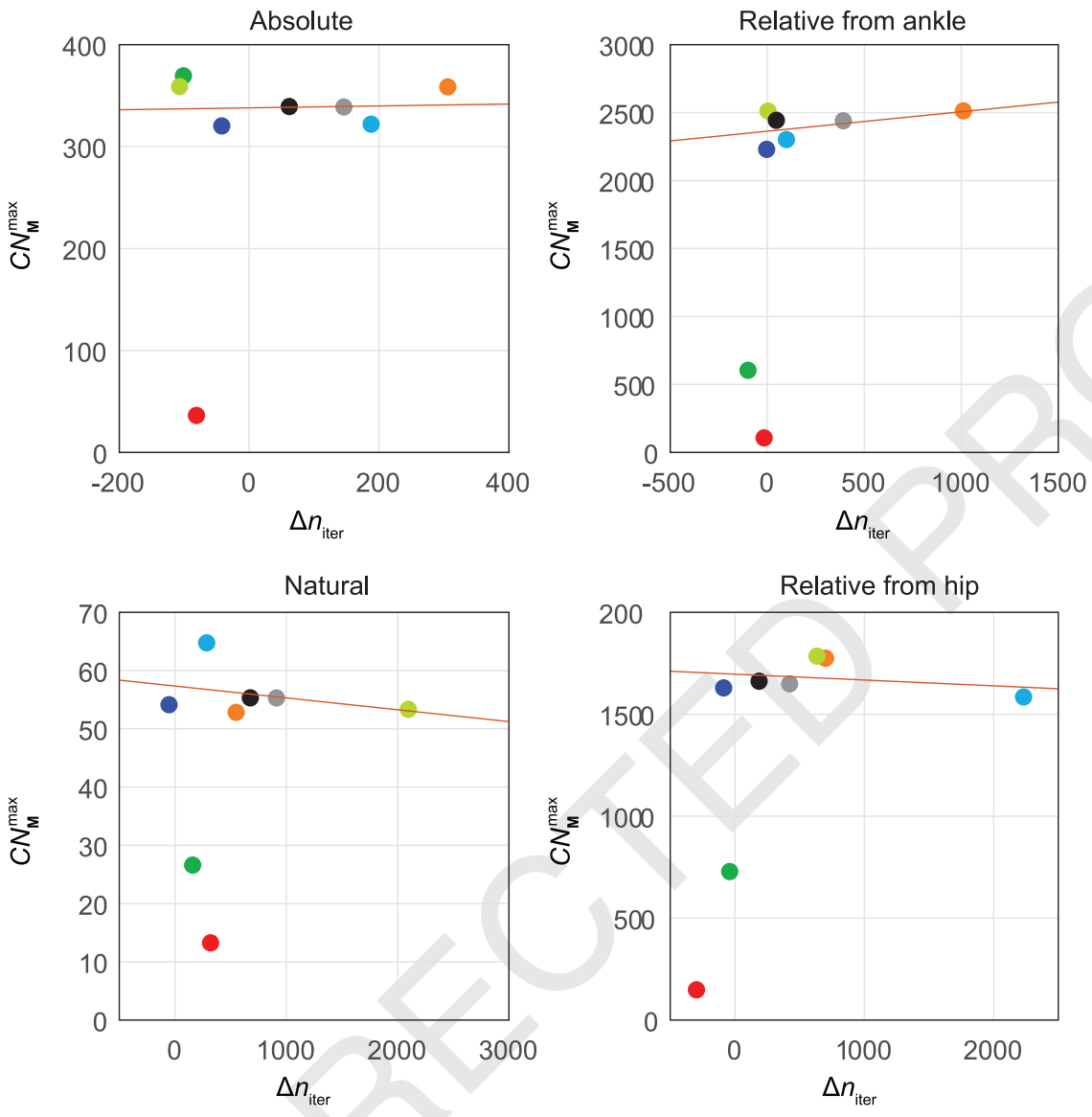

$3 \mathrm{DoF} \bullet 4 \mathrm{DoF} \bullet 5 \mathrm{DoF} \bigcirc 6 \mathrm{DoF}$

$7 \mathrm{DoF}$

$8 \mathrm{DoF} \bigcirc 9 \mathrm{DoF}$

$10 \mathrm{DoF}$

Fig. 4. Maximum condition number $\left(\mathrm{CN}_{\mathrm{M}}^{\max }\right)$ with respect to the difference in number of iterations $\left(\Delta n_{\text {iter }}\right)$ between implicit and explicit formulations for each type of coordinate. The red lines represent the regression in models from 5 to 10 DoF. Notice that horizontal and vertical axes do not have the same scale in each graph.

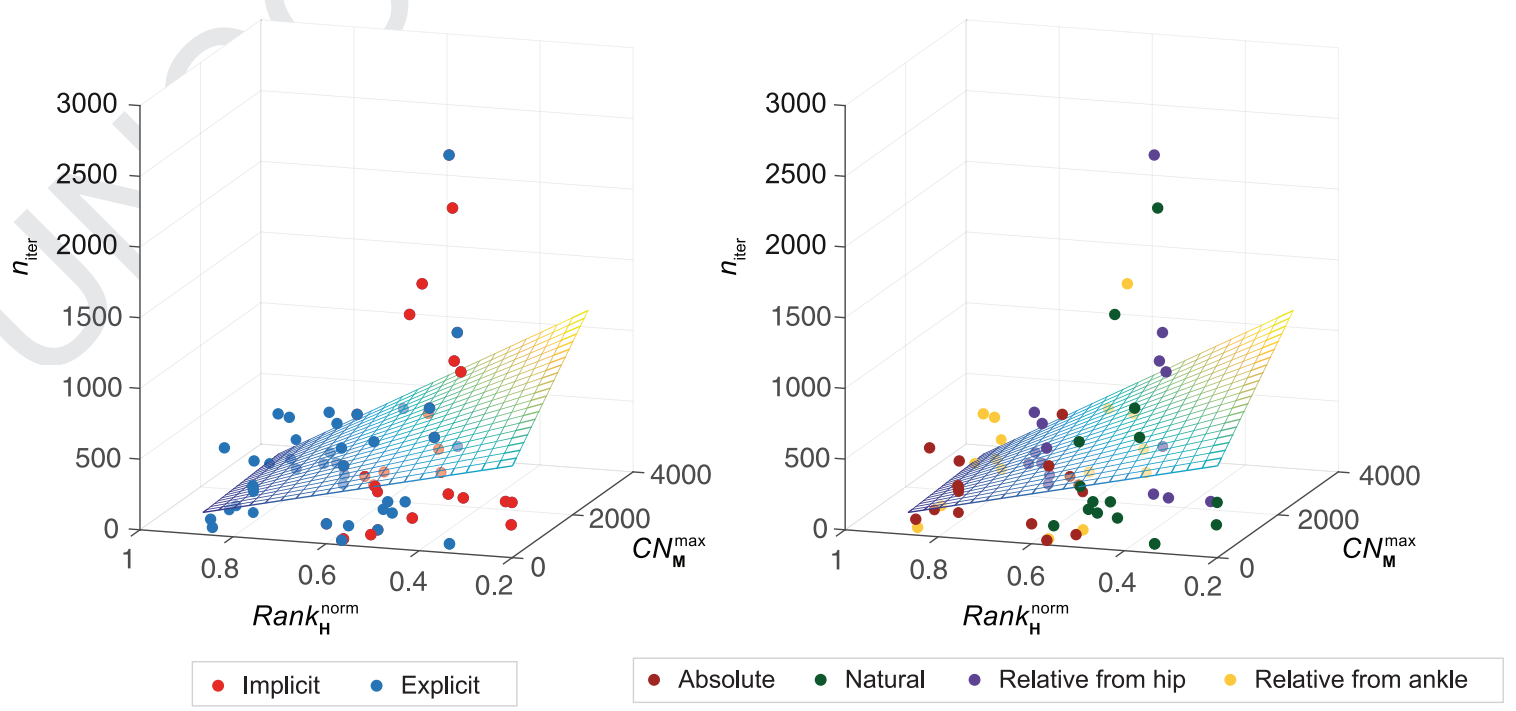

(a)

(b)

Fig. 5. Number of iterations $\left(n_{i \text { ter }}\right)$ with respect to normalized rank of the Hessian (Rank $k_{\mathrm{H}}^{\text {norm }}$ ) and the maximum condition number of the mass matrix $\left(C N_{\mathrm{M}}^{\max }\right)$. The surface represents the best-fit plane for all points. (a) Comparison by explicit and implicit formulations. (b) Comparison by type of coordinates. 
number of iterations is not explained only by the condition number of the mass matrix of the multibody system. Instead, it depends on the condition number of the mass matrix and the rank of the Hessian of the NLP problem simultaneously.

The formulations using absolute coordinates are the ones with the lowest condition number of the mass matrix and the highest rank of the Hessian of the NLP problem, so they are also the ones that require the lowest number of iterations.

Relative coordinates starting the kinematic chain from the hip lead to higher condition number values of the mass matrix compared to the ones starting the kinematics from the ankle. Therefore, we would expect to obtain a higher number of iterations to find an optimal solution with the model starting the kinematic chain from the hip. However, this formulation also leads to higher ranks of the Hessian matrices of the NLP problem. Hence, there is no systematic tendency between the number of iterations of both formulations, which suggests that there is a balance between high values of the rank of the NLP Hessian and high values of the condition number of mass matrices.

Multibody models formulated with natural coordinates have constant mass matrices and a smaller condition number compared to the other formulations. However, optimal control problems formulated with natural coordinates do not lead to faster convergences (lower number of iterations) than the other formulations with no constant mass matrices. This result can be due to the lower rank of the Hessian of the NLP problem obtained using this formulation compared to the other formulations.

The type of dynamic formulation (explicit or implicit) affects the rank of the Hessian matrix. This is due to the fact that the introduction of acceleration controls as design variables increases the dimension of the Hessian. In turn, since this study does not include these variables in the cost function -they appear only in linear form in the constraint function-, the columns increased in the Hessian matrix contain only zeros. Therefore, the rank of the matrix decreases. This decrease seems to be the reason why explicit formulations usually converge with a lower number of iterations than the implicit ones with more complex models (number of DoFs $>5$ ). Including the controls in the cost function -when implicit formulations are used-could help to obtain better convergences (as in Meyer et al. [21] or De Groote et al. [6]) though then the objective criteria is slightly modified.

Those conclusions are in line with other studies. For example, Leger and McPhee [42] developed a heuristic method to select the most appropriate set of coordinates for a multibody forward dynamics analysis. The study suggested that minimizing the number of modeling coordinates of the multibody system does not always produce simulations with faster convergence. De Groote et al. [6] also found that the minimal number of design variables did not always lead to the fastest convergence. The novel contribution of our study is two-fold. On the one hand, we provide the comparison of the number of iterations among the different tested formulations when solving a typical biomechanical optimal control problem. On the other hand, we found a significant statistical relation among the number of iterations needed to solve the problem, and the maximum condition number of the mass matrix and the normalized rank of the NLP Hessian.

The presented work has some limitations. Firstly, the results are only obtained using planar models. However, we would expect similar results using three dimensional models. This relies on the fact that the variations in the condition of mass matrices or the changes in Hessian matrices among formulations obtained with three dimensional models would be similar to the ones obtained in planar models. Secondly, our biomechanical models have one ankle attached to the ground, whereas normally in biomechanical problems, contact models are used to model the ground reaction forces. However, the type of contact model [43] could also have an influence on the convergence, which we choose to leave out of the scope of the study. Finally, different formulations of the cost function or constraints could influence the rank of the NLP Hessian and, in turn, affect the performance of the optimization. Nevertheless, the goal of this study was to analyze the effect of the type of coordinates in a representative formulation of a biomechanical optimal control problem.

This study focuses on the analysis of the numerical convergence of the optimal control problems, and not on the computational time spent to solve the optimizations, which is also an important factor. Although it is known that explicit formulations will take more time per iteration than implicit formulations (due to the need to invert the mass matrix [29]), the analysis on the computational time was out of the scope of this study. This was due to the fact that the computational time can depend on the type of the CPU or the method used to evaluate the functions. Future work could be focused on analyzing this variable.

In conclusion, the main message of the presented work is that, in order to formulate a biomechanical optimal control problem, one should care about the type of coordinates and the dynamic formulation being used. We showed that, since the coordinates and the dynamic formulation affect the condition number of the mass matrix and the rank of the NLP Hessian, these directly affect the convergence behavior of the optimization problem.

\section{Supplementary materials}

Supplementary material associated with this article can be found, in the online version, at doi:10.1016/j.mechmachtheory. 2019.103578.

\section{Appendix A}

Fig. A1

Table A1 and A2 
Table A1

Number of discretized state and control variables, and constraint multipliers of the multibody Lagrange equations for each model and formulation of the optimal control problem.

\begin{tabular}{|c|c|c|c|c|c|}
\hline Model & Coordinate type & $\begin{array}{l}\text { Explicit (E) / } \\
\text { Implicit (I) }\end{array}$ & $\begin{array}{l}\text { Number of disc. } \\
\text { state var. }\end{array}$ & $\begin{array}{l}\text { Number of disc. } \\
\text { control var. }\end{array}$ & $\begin{array}{l}\text { Number of disc. } \\
\text { constraint multipliers }\end{array}$ \\
\hline \multirow[t]{8}{*}{$3 \mathrm{DoF}$} & Absolute & E & 2406 & 303 & 0 \\
\hline & & I & 2406 & 606 & 0 \\
\hline & Relative from ankle & $\mathrm{E}$ & 2046 & 303 & 0 \\
\hline & & I & 2046 & 606 & 0 \\
\hline & Relative from hip & $\mathrm{E}$ & 4010 & 303 & 202 \\
\hline & & I & 4010 & 808 & 202 \\
\hline & Natural & E & 4812 & 303 & 303 \\
\hline & & I & 4812 & 909 & 303 \\
\hline \multirow[t]{8}{*}{$4 \mathrm{DoF}$} & Absolute & E & 3208 & 404 & 0 \\
\hline & & I & 3208 & 808 & 0 \\
\hline & Relative from ankle & E & 3208 & 404 & 0 \\
\hline & & I & 3208 & 808 & 0 \\
\hline & Relative from hip & $\mathrm{E}$ & 4812 & 404 & 202 \\
\hline & & I & 4812 & 1010 & 202 \\
\hline & Natural & E & 6416 & 404 & 404 \\
\hline & & I & 6416 & 1212 & 404 \\
\hline \multirow[t]{8}{*}{5 DoF } & Absolute & E & 4010 & 505 & 0 \\
\hline & & I & 4010 & 1010 & 0 \\
\hline & Relative from ankle & E & 4010 & 505 & 0 \\
\hline & & I & 4010 & 1010 & 0 \\
\hline & Relative from hip & $\mathrm{E}$ & 5614 & 505 & 202 \\
\hline & & I & 5614 & 1212 & 202 \\
\hline & Natural & $\mathrm{E}$ & 8020 & 505 & 1010 \\
\hline & & I & 8020 & 1515 & 1010 \\
\hline \multirow[t]{8}{*}{6 DoF } & Absolute & E & 9612 & 1206 & 0 \\
\hline & & I & 9612 & 2412 & 0 \\
\hline & Relative from ankle & $\mathrm{E}$ & 9612 & 1206 & 0 \\
\hline & & I & 9612 & 2412 & 0 \\
\hline & Relative from hip & E & 12,816 & 1206 & 402 \\
\hline & & I & 12,816 & 2814 & 402 \\
\hline & Natural & $\mathrm{E}$ & 19,224 & 1206 & 1206 \\
\hline & & I & 19,224 & 3618 & 1206 \\
\hline \multirow[t]{8}{*}{$7 \mathrm{DoF}$} & Absolute & E & 22,414 & 2807 & 0 \\
\hline & & I & 22,414 & 5614 & 0 \\
\hline & Relative from ankle & E & 22,414 & 2807 & 0 \\
\hline & & I & 22,414 & 5614 & 0 \\
\hline & Relative from hip & E & 28,818 & 2807 & 802 \\
\hline & & I & 28,818 & 6416 & 802 \\
\hline & Natural & $\mathrm{E}$ & 44,828 & 2807 & 2807 \\
\hline & & I & 44,828 & 8421 & 2807 \\
\hline \multirow[t]{8}{*}{8 DoF } & Absolute & E & 25,616 & 3208 & 0 \\
\hline & & I & 25,616 & 6416 & 0 \\
\hline & Relative from ankle & E & 25,616 & 3208 & 0 \\
\hline & & I & 25,616 & 6416 & 0 \\
\hline & Relative from hip & $\mathrm{E}$ & 32,020 & 3208 & 802 \\
\hline & & I & 32,020 & 7218 & 802 \\
\hline & Natural & $\mathrm{E}$ & 51,232 & 3208 & 3208 \\
\hline & & I & 51,232 & 9624 & 3208 \\
\hline \multirow[t]{8}{*}{$9 \mathrm{DoF}$} & Absolute & $\mathrm{E}$ & 14,418 & 1809 & 0 \\
\hline & & I & 14,418 & 3618 & 0 \\
\hline & Relative from ankle & $\mathrm{E}$ & 14,418 & 1809 & 0 \\
\hline & & I & 14,418 & 3618 & 0 \\
\hline & Relative from hip & E & 17,622 & 1809 & 402 \\
\hline & & I & 17,622 & 4020 & 402 \\
\hline & Natural & E & 28,836 & 1809 & 1809 \\
\hline & & I & 28,836 & 5427 & 1809 \\
\hline \multirow[t]{8}{*}{$10 \mathrm{DoF}$} & Absolute & E & 16,020 & 2010 & 0 \\
\hline & & I & 16,020 & 4020 & 0 \\
\hline & Relative from ankle & E & 16,020 & 2010 & 0 \\
\hline & & I & 16,020 & 4020 & 0 \\
\hline & Relative from hip & $\mathrm{E}$ & 19,224 & 2010 & 402 \\
\hline & & I & 19,224 & 4422 & 402 \\
\hline & Natural & E & 32,040 & 2010 & 2010 \\
\hline & & I & 32,040 & 6030 & 2010 \\
\hline
\end{tabular}


Table A2

Outcomes of all optimal control problems. Cost function value, number of iterations, maximum condition number of the mass matrix ( $\mathrm{CN}_{\mathbf{M}}$ max $)$, rank of the NLP Hessian $\left(\operatorname{Ran}_{\mathbf{H}}\right)$, normalized rank of the Hessian, which is the quotient between the rank of the Hessian and the total number of rows of the Hessian $\left(\operatorname{Rank}_{\mathbf{H}}^{\text {norm }}\right)$, and mean \pm standard deviation of root mean square difference between the Cartesian coordinates obtained for each formulation with respect to the one from absolute explicit formulation ( $\left.r m s^{\text {coord }}\right)$.

\begin{tabular}{|c|c|c|c|c|c|c|c|c|}
\hline Model & Coordinate type & $\begin{array}{l}\text { Explicit } \\
\text { (E)/Implicit (I) }\end{array}$ & $\begin{array}{l}\text { Cost function } \\
{\left[\mathrm{N}^{2} \mathrm{~m}^{2}{ }^{0} \cdot 10^{4}\right]}\end{array}$ & $\begin{array}{l}\text { number } \\
\text { iterations }\end{array}$ & $C N_{\mathbf{M}}^{\max }$ & $\operatorname{Rank}_{\mathbf{H}}$ & $\operatorname{Rank}_{\mathbf{H}}^{\text {norm }}$ & $r m s^{\mathrm{coord}}[\mathrm{mm}]$ \\
\hline \multirow[t]{8}{*}{$3 \mathrm{DoF}$} & \multirow[t]{2}{*}{ Absolute } & $\mathrm{E}$ & 8.75 & 103 & 35.5 & 2301 & 0.85 & - \\
\hline & & I & 8.75 & 23 & 35.5 & 1710 & 0.57 & $0.001 \pm 0.001$ \\
\hline & \multirow[t]{2}{*}{ Relative from ankle } & $\mathrm{E}$ & 8.75 & 35 & 101.1 & 2302 & 0.85 & $0.0008 \pm 0.008$ \\
\hline & & I & 8.75 & 23 & 101.1 & 1711 & 0.57 & $0.001 \pm 0.001$ \\
\hline & \multirow[t]{2}{*}{ Relative from hip } & $\mathrm{E}$ & 8.74 & 657 & 144.2 & 2597 & 0.58 & $0.2 \pm 0.1$ \\
\hline & & I & 8.75 & 366 & 144.2 & 1117 & 0.22 & $0.3 \pm 0.2$ \\
\hline & \multirow[t]{2}{*}{ Natural } & $\mathrm{E}$ & 8.75 & 58 & 13.1 & 1812 & 0.33 & $0.05 \pm 0.02$ \\
\hline & & I & 8.75 & 385 & 13.1 & 1206 & 0.20 & $0.06 \pm 0.02$ \\
\hline \multirow[t]{8}{*}{$4 \mathrm{DoF}$} & \multirow[t]{2}{*}{ Absolute } & $\mathrm{E}$ & 8.47 & 123 & 368.7 & 3002 & 0.83 & - \\
\hline & & I & 8.47 & 23 & 368.7 & 2115 & 0.53 & $0.07 \pm 0.01$ \\
\hline & \multirow[t]{2}{*}{ Relative from ankle } & $\mathrm{E}$ & 8.47 & 117 & 598.3 & 3002 & 0.83 & $0.004 \pm 0.004$ \\
\hline & & $\mathrm{I}$ & 8.47 & 22 & 598.3 & 2115 & 0.53 & $0.07 \pm 0.1$ \\
\hline & \multirow[t]{2}{*}{ Relative from hip } & $\mathrm{E}$ & 8.48 & 306 & 724.6 & 3302 & 0.61 & $0.7 \pm 1.0$ \\
\hline & & $\mathrm{I}$ & 8.48 & 272 & 724.8 & 2115 & 0.35 & $2 \pm 4$ \\
\hline & \multirow[t]{2}{*}{ Natural } & $\mathrm{E}$ & 8.47 & 58 & 26.5 & 2424 & 0.34 & $0.04 \pm 0.02$ \\
\hline & & I & 8.47 & 224 & 26.5 & 1628 & 0.20 & $0.004 \pm 0.001$ \\
\hline \multirow[t]{8}{*}{$5 \mathrm{DoF}$} & \multirow[t]{2}{*}{ Absolute } & $\mathrm{E}$ & 1.23 & 125 & 319.5 & 3505 & 0.78 & - \\
\hline & & I & 1.23 & 84 & 319.5 & 3107 & 0.62 & $0.04 \pm 0.06$ \\
\hline & Relative from ankle & $\mathrm{E}$ & 1.23 & 161 & 2224.1 & 3905 & 0.86 & $0.01 \pm 0.01$ \\
\hline & & $\mathrm{I}$ & 1.23 & 162 & 2224.1 & 3107 & 0.62 & $0.04 \pm 0.06$ \\
\hline & Relative from hip & $\mathrm{E}$ & 1.23 & 221 & 1625.3 & 4204 & 0.67 & $0.2 \pm 0.2$ \\
\hline & & $\mathrm{I}$ & 1.23 & 140 & 1625.3 & 3105 & 0.44 & $0.2 \pm 0.2$ \\
\hline & Natural & $\mathrm{E}$ & 1.23 & 261 & 54.0 & 4572 & 0.48 & $0.04 \pm 0.02$ \\
\hline & & I & 1.23 & 215 & 54.0 & 4398 & 0.42 & $0.04 \pm 0.02$ \\
\hline $6 \mathrm{DoF}$ & Absolute & $\mathrm{E}$ & 1.24 & 314 & 321.2 & 16,806 & 0.78 & - \\
\hline & & I & 1.24 & 504 & 321.2 & 13,984 & 0.58 & $0.003 \pm 0.003$ \\
\hline & Relative from ankle & $\mathrm{E}$ & 1.24 & 505 & 2296.1 & 18,402 & 0.85 & $0.002 \pm 0.003$ \\
\hline & & $\mathrm{I}$ & 1.24 & 608 & 2296.1 & 13,984 & 0.58 & $0.003 \pm 0.003$ \\
\hline & Relative from hip & $\mathrm{E}$ & 1.24 & 308 & 1580.9 & 19,576 & 0.68 & $0.01 \pm 0.02$ \\
\hline & & $\mathrm{I}$ & 1.24 & 2546 & 1580.9 & 14,006 & 0.44 & $0.03 \pm 0.05$ \\
\hline & Natural & $\mathrm{E}$ & 1.24 & 125 & 64.6 & 23,986 & 0.55 & $0.02 \pm 0.02$ \\
\hline & & I & 1.24 & 417 & 64.6 & 23,913 & 0.50 & $0.002 \pm 0.002$ \\
\hline 7 DoF & Absolute & $\mathrm{E}$ & 1.47 & 561 & 357.9 & 21,203 & 0.84 & - \\
\hline & & I & 1.47 & 868 & 357.9 & 15,566 & 0.56 & $0.01 \pm 0.02$ \\
\hline & Relative from ankle & $\mathrm{E}$ & 1.47 & 451 & 2506.9 & 21,207 & 0.84 & $0.001 \pm 0.002$ \\
\hline & & I & 1.47 & 1466 & 2506.9 & 15,565 & 0.56 & $0.01 \pm 0.02$ \\
\hline & Relative from hip & $\mathrm{E}$ & 1.47 & 556 & 1771.0 & 22,391 & 0.69 & $0.01 \pm 0.02$ \\
\hline & & I & 1.47 & 1263 & 1771.0 & 15,564 & 0.43 & $0.01 \pm 0.02$ \\
\hline & Natural & $\mathrm{E}$ & 1.47 & 240 & 52.7 & 23,214 & 0.46 & $2 \pm 3$ \\
\hline & & I & 1.47 & 797 & 52.7 & 20,716 & 0.37 & $0.008 \pm 0.02$ \\
\hline $8 \mathrm{DoF}$ & Absolute & $\mathrm{E}$ & 1.28 & 290 & 338.1 & 22,408 & 0.78 & - \\
\hline & & I & 1.28 & 437 & 338.1 & 17,219 & 0.54 & $0.003 \pm 0.004$ \\
\hline & Relative from ankle & $\mathrm{E}$ & 1.28 & 168 & 2434.2 & 24,008 & 0.83 & $0.002 \pm 0.003$ \\
\hline & & I & 1.28 & 564 & 2434.2 & 17,217 & 0.54 & $0.003 \pm 0.004$ \\
\hline & Relative from hip & $\mathrm{E}$ & 1.28 & 652 & 1645.3 & 25,193 & 0.70 & $0.007 \pm 0.01$ \\
\hline & & I & 1.28 & 1081 & 1645.3 & 17,235 & 0.43 & $0.5 \pm 1.0$ \\
\hline & Natural & $\mathrm{E}$ & 1.28 & 734 & 55.2 & 28,802 & 0.50 & $0.6 \pm 1.1$ \\
\hline & & I & 1.28 & 1653 & 55.2 & 27,101 & 0.42 & $0.003 \pm 0.003$ \\
\hline 9 DoF & Absolute & $\mathrm{E}$ & 1.47 & 484 & 358.2 & 12,609 & 0.78 & - \\
\hline & & I & 1.47 & 378 & 358.2 & 9378 & 0.52 & $0.01 \pm 0.02$ \\
\hline & Relative from ankle & $\mathrm{E}$ & 1.47 & 297 & 2505.7 & 13,410 & 0.83 & $0.01 \pm 0.03$ \\
\hline & & I & 1.47 & 305 & 2505.7 & 9377 & 0.52 & $0.01 \pm 0.02$ \\
\hline & Relative from hip & $\mathrm{E}$ & 1.47 & 344 & 1781.1 & 14,000 & 0.71 & $0.02 \pm 0.04$ \\
\hline & & $\mathrm{I}$ & 1.47 & 985 & 1781.1 & 9367 & 0.42 & $0.03 \pm 0.04$ \\
\hline & Natural & $\mathrm{E}$ & 1.47 & 326 & 53.2 & 14,030 & 0.43 & $0.05 \pm 0.04$ \\
\hline & & I & 1.47 & 2429 & 53.2 & 11,927 & 0.33 & $0.08 \pm 0.07$ \\
\hline $10 \mathrm{DoF}$ & Absolute & $\mathrm{E}$ & 1.28 & 271 & 338.5 & 14,010 & 0.78 & - \\
\hline & & I & 1.28 & 334 & 338.5 & 10,227 & 0.51 & $0.007 \pm 0.01$ \\
\hline & Relative from ankle & $\mathrm{E}$ & 1.28 & 102 & 2437.8 & 14,810 & 0.82 & $0.002 \pm 0.003$ \\
\hline & & $\mathrm{I}$ & 1.28 & 153 & 2437.8 & 10,226 & 0.51 & $0.007 \pm 0.01$ \\
\hline & Relative from hip & $\mathrm{E}$ & 1.28 & 262 & 1658.1 & 15,406 & 0.71 & $0.02 \pm 0.03$ \\
\hline & & $\mathrm{I}$ & 1.28 & 612 & 1659.4 & 10,198 & 0.42 & $0.03 \pm 0.04$ \\
\hline & Natural & $\mathrm{E}$ & 1.28 & 317 & 55.2 & 16,827 & 0.47 & $0.04 \pm 0.04$ \\
\hline & & $\mathrm{I}$ & 1.28 & 1001 & 55.2 & 15,238 & 0.38 & $0.005 \pm 0.004$ \\
\hline
\end{tabular}



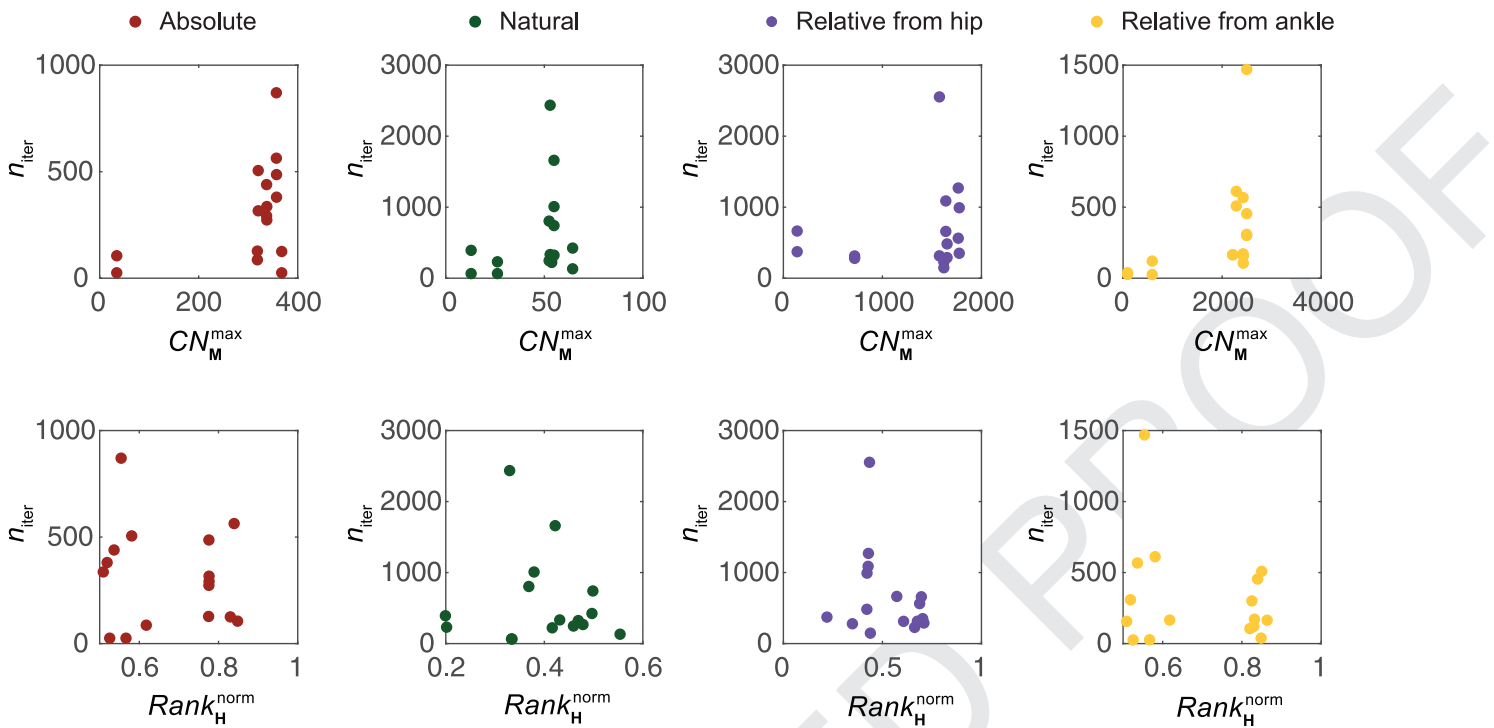

Fig. A1. Number of iterations with respect to the maximum of the condition number (upper row) and with respect to the normalized rank of the Hessian (lower row) for each type of coordinate. Note that the scale of the plots are not the same.

\section{References}

[1] R. Fluit, M.M. van der Krogt, H. van der Kooij, N. Verdonschot, H.F.J.M. Koopman, A simple controller for the prediction of three-dimensional gait, J. Biomech. 45 (15) (2012) 2610-2617, doi:10.1016/j.jbiomech.2012.08.019.

[2] M. Sreenivasa, M. Millard, P. Manns, K. Mombaur, Optimizing wearable assistive devices with neuromuscular models and optimal Control, in: N Ibáñez, J., Al., Ed. Converging Clin. Eng. Res. Neurorehabilitation II. Proc. 3rd Int. Conf. Neurorehabilitation, n.d. pp. 627-632.

[3] N.P. Fey, G.K. Klute, R.R. Neptune, Optimization of prosthetic foot stiffness to reduce metabolic cost and intact knee loading during below-knee amputee walking: a theoretical study, J. Biomech. Eng. 134 (11) (2012) 111005, doi:10.1115/1.4007824.

[4] M. Millard, E. Kubica, J.J. McPhee, Forward dynamic human gait simulation using a SLIP target model, Procedia IUTAM 2 (2011) 142-157, doi:10.1016/ j.piutam.2011.04.015.

[5] K. Mombaur, Optimal control for applications in medical and rehabilitation technology: challenges and solutions, in: Adv. Math. Model. Optim. Optim. Control (2016) 103-145, doi:10.1007/978-3-319-30785-5.

[6] F. De Groote, A.L. Kinney, A.V. Rao, B.J. Fregly, Evaluation of direct collocation optimal control problem formulations for solving the muscle redundancy problem, Ann. Biomed. Eng 44 (10) (2016) 2922-2936, doi:10.1007/s10439-016-1591-9.

[7] L.L. Menegaldo, A. de, T. Fleury, H.I. Weber, A "cheap" optimal control approach to estimate muscle forces in musculoskeletal systems, J. Biomech 39 (2006) 1787-1795, doi:10.1016/j.jiomech.2005.05.029.

[8] G. Bessonnet, P. Seguin, P. Sardain, A parametric optimization approach to walking pattern synthesis, Int. J. Rob. Res. 24 (7) (2005) 523-536. https: //doi.org/10.1177/0278364905055377.

[9] L. Ren, R.K. Jones, D. Howard, Predictive modelling of human walking over a complete gait cycle, J. Biomech 40 (7) (2007) 1567-1574, doi:10.1016/j. jbiomech.2006.07.017.

[10] Y. Xiang, J.S. Arora, S. Rahmatalla, K. Abdel-malek, Optimization-based dynamic human walking prediction: one step formulation, Int. J. Numer. Methods Eng 79 (6) (2009) 667-695, doi:10.1002/nme.

[11] L.L. Menegaldo, A.D.T. Fleury, H.I. Weber, Biomechanical modeling and optimal control of human posture, J. Biomech 36 (11) (2003) 1701-1712, doi:10. 1016/S0021-9290(03)00170-2.

[12] F.C. Anderson, M.G. Pandy, Dynamic optimization of human walking, j, Biomech. Eng. 123 (2001) 381-390, doi:10.1115/1.1392310.

[13] M. Ackermann, A.J. Van den Bogert, Optimality principles for model-based prediction of human gait, J. Biomech 43 (6) (2010) 1055-1060, doi:10.1177/ 1464419313488363.

[14] M.S. Shourijeh, J. McPhee, Forward dynamic optimization of human gait simulations: a global parameterization approach, J. Comput. Nonlinear Dyn. 9 (3) (2014) $031018031018-03018-11$, doi:10.1115/1.4026266.

[15] M.L. Kaplan, J.H. Heegaard, Predictive algorithms for neuromuscular control of human locomotion, J. Biomech 34 (8) (2001) 1077-1083.

[16] T.W. Dorn, J.M. Wang, J.L. Hicks, S. Delp, Predictive simulation generates human adaptations during loaded and inclined walking, PLoS ONE 10 (4) (2015) 1-16, doi:10.1371/journal.pone.0121407.

[17] S. Porsa, Y.C. Lin, M.G. Pandy, Direct methods for predicting movement biomechanics based upon optimal control theory with implementation in opensim, Ann. Biomed. Eng 44 (8) (2016) 2542-2557, doi:10.1007/s10439-015-1538-6.

[18] A. Falisse, G. Serrancoli, C. Dembia, S. Song, I. Jonkers, F. De Groote, Computationally efficient predictive muscle-driven simulations of 3D walking,, 8th world congr. Biomech., 2018.

[19] L.-.F. Lee, B.R. Umberger, Generating optimal control simulations of musculoskeletal movement using opensim and MATLAB, PeerJ. 4 (2016) 1-18, doi: $10.7717 /$ peerj.1638.

[20] D. García-Vallejo, W. Schiehlen, 3D-Simulation of human walking by parameter optimization, Arch. Appl. Mech 82 (4) (2012) 533-556, doi:10.1007/ s00419-011-0571-7.

[21] A.J. Meyer, I. Eskinazi, J.N. Jackson, A.V. Rao, C. Patten, B.J. Fregly, Muscle synergies facilitate computational prediction of subject-specific walking motions, Front. Bioeng. Biotechnol 4 (2016) 77, doi:10.3389/fbioe.2016.00077.

[22] G. Serrancolí, A. Falisse, C. Dembia, J. De Schutter, F. De Groote, Estimation of human-exoskeleton collaborative movement, World Congr. Biomech, 2018.

[23] Y. Xiang, J.S. Arora, K. Abdel-Malek, Hybrid predictive dynamics: a new approach to simulate human motion, Multibody Syst. Dyn 28 (3) (2012) 199-224, doi:10.1007/s11044-012-9306-y. 
[24] L.S. Jennings, K.H. Wong, K.L. Teo, An optimal control problem in biomechanics, in: IFAC World Congr. 12th Trienn. Wold Congr. Int. Fed. Autom. Control., Elsevier, 1993, pp. 279-282, doi:10.1155/S016117129100073X.

[25] R. Maas, S. Leyendecker, Biomechanical optimal control of human arm motion, Proc. Inst. Mech. Eng. Part K J. Multi-Body Dyn 227 (4) (2013) 375-389, doi:10.1177/1464419313488363.

[26] M.G. Pandy, F.C. Anderson, D.G. Hull, A parameter optimization approach for the optimal control of large-scale musculoskeletal systems, J. Biomech. Eng. 114 (4) (1992) 450-460, doi:10.1115/1.2894094.

[27] J.T. Betts, Practical methods for optimal control and estimation using nonlinear programming, 2010. doi:10.1137/1.9780898718577.

[28] P. Betsch, R. Siebert, N. Sänger, Natural coordinates in the optimal control of multibody systems, J. Comput. Nonlinear Dyn 7 (1) (2012) 011009 , doi:10.1115/1.4004886.

[29] A.J. Van Den Bogert, D. Blana, D. Heinrich, Implicit methods for efficient musculoskeletal simulation and optimal control, Procedia IUTAM 2 (2011) 297-316, doi:10.1016/j.piutam.2011.04.027.

[30] Y.C. Lin, M.G. Pandy, Three-dimensional data-tracking dynamic optimization simulations of human locomotion generated by direct collocation, J. Biomech. 59 (2017) 1-8, doi:10.1016/j.jbiomech.2017.04.038.

[31] J.G. De Jalón, M.D. Gutiérrez-López, Multibody dynamics with redundant constraints and singular mass matrix: existence, uniqueness, and determination of solutions for accelerations and constraint forces, Multibody Syst. Dyn. 30 (2013) (2013) 311-341, doi:10.1007/s11044-013-9358-7.

[32] J.G. De Jalón, Twenty-five years of natural coordinates, Multibody Syst. Dyn. 18 (1) (2007) 15-33, doi:10.1007/s11044-007-9068-0.

[33] A.K. Cline, C.B. Moler, S.G. Stewart, J.H. Wilkinson, An estimate for the condition number of a matrix, SIAM J. Numer. Anal. 16 (2) (1979) $368-375$.

[34] R. Serban, J.S. Freeman, Identification and identifiability of unknown parameters in multibody dynamic systems, Multibody Syst. Dyn. 5 (2001) 335350, doi:10.1023/A:1011434711375.

[35] Y. Kang, T.W. Lin, Y.J. Chang, Y.P. Chang, C.C. Wang, Optimal balancing of flexible rotors by minimizing the condition number of influence coefficients, Mech. Mach. Theory 43 (7) (2008) 891-908, doi:10.1016/j.mechmachtheory.2007.06.005.

[36] J.J. McPhee, S.M. Redmond, Modelling multibody systems with indirect coordinates, Comput. Methods Appl. Mech. Eng. 195 (50-51) (2006) 6942-6957, doi:10.1016/j.cma.2005.02.033.

[37] J.G. De Jalón, E. Bayo, Kinematic and Dynamic Simulation of Multibody systems: the Real-Time Challenge, Springer-Verlag, New York, 1994.

[38] M. Da Lio, V. Cossalter, R. Lot, On the use of natural coordinates in optimal synthesis of mechanisms, Mech. Mach. Theory 35 (10) (2000) 1367-1389, doi:10.1016/S0094-114X(00)00006-9.

[39] D.A. Benson, G.T. Huntington, T.P. Thorvaldsen, A.V. Rao, Direct trajectory optimization and costate estimation via an orthogonal collocation method, J. Guid. Control. Dyn. 29 (2006) 1435-1440, doi:10.2514/1.20478.

[40] J.A.E. Andersson, J. Gillis, G. Horn, J.B. Rawlings, M. Diehl, CasADi: a software framework for nonlinear optimization and optimal control, Math. Program. Comput. 11 (1) (2018) 1-36, doi:10.1007/s12532-018-0139-4.

[41] A. Wächter, L.T. Biegler, On the implementation of an interior-point filter line-search algorithm for large-scale nonlinear programming, Math Progr. 106 (1) (2006) 25-57, doi:10.1007/s10107-004-0559-y.

[42] M. Léger, J. McPhee, Selection of modeling coordinates for forward dynamic multibody simulations, Multibody Syst. Dyn. 18 (2) (2007) 277-297, doi:10.1007/s11044-007-9083-1.

[43] M. Machado, P. Moreira, P. Flores, H. Lankarani, Compliant contact force models in multibody dynamics: evolution of the Hertz contact theory, Mech. Mach. Theory 53 (2012) 99-121. 